\title{
Measuring Players' Experience of Need Satisfaction in Digital Games: An Analysis of the Factor Structure of the UPEQ
}

\author{
Dominik Kayser \\ dominik.kayser@unibas.ch \\ Center for General Psychology and \\ Methodology, Faculty of Psychology, \\ University of Basel \\ Basel, Switzerland
}

\author{
Sebastian A. C. Perrig \\ sebastian.perrig@unibas.ch \\ Center for General Psychology and \\ Methodology, Faculty of Psychology, \\ University of Basel \\ Basel, Switzerland
}

\author{
Florian Brühlmann \\ florian.bruehlmann@unibas.ch \\ Center for General Psychology and \\ Methodology, Faculty of Psychology, \\ University of Basel \\ Basel, Switzerland
}

\begin{abstract}
In this work, we explore the factorial structure of the Ubisoft Perceived Experience Questionnaire (UPEQ) and its correlation with game enjoyment. For this purpose, an online survey was conducted on the experience with the video game League of Legends. Three hundred and sixty-nine participants provided information about their in- and out-of-game demographics and rated their experience with the game using the UPEQ and the subscale Interest/Enjoyment from the Intrinsic Motivation Inventory. Using confirmatory and exploratory factor analysis, we found weaknesses in the 3-factor model of the UPEQ and propose a 6- or 7-factor structure as a basis for new research and improvement of the UPEQ. All materials are available on OSF: https://osf.io/6nhts/.
\end{abstract}

\section{CCS CONCEPTS}

- Human-centered computing $\rightarrow$ Empirical studies in $\mathrm{HCI}$; HCI theory, concepts and models; • Applied computing $\rightarrow$ Computer games; • Information systems $\rightarrow$ Massively multiplayer online games.

\section{KEYWORDS}

Video Games; Player Experience; Factor Analysis; Questionnaire; Validation; League of Legends; UPEQ

\section{ACM Reference Format:}

Dominik Kayser, Sebastian A. C. Perrig, and Florian Brühlmann. 2021. Measuring Players' Experience of Need Satisfaction in Digital Games: An Analysis of the Factor Structure of the UPEQ. In Extended Abstracts of the 2021 Annual Symposium on Computer-Human Interaction in Play (CHI PLAY '21), October 18-21, 2021, Virtual Event, Austria. ACM, New York, NY, USA, 5 pages. https://doi.org/10.1145/3450337.3483499

\section{INTRODUCTION}

In recent years, digital games have become a mainstream source of entertainment and leisure. Digital games advanced from being simply a way of passing the time to a medium that can make people feel happy, sad, angry, and disgusted [11]. With the emergence of different genres and types of digital games, there has been a

Permission to make digital or hard copies of part or all of this work for personal or classroom use is granted without fee provided that copies are not made or distributed for profit or commercial advantage and that copies bear this notice and the full citation on the first page. Copyrights for third-party components of this work must be honored

For all other uses, contact the owner/author(s).

CHI PLAY '21, October 18-21, 2021, Virtual Event, Austria

(c) 2021 Copyright held by the owner/author(s).

ACM ISBN 978-1-4503-8356-1/21/10.

https://doi.org/10.1145/3450337.3483499 growing interest in assessing people's experiences playing these games. But while there exists an uncountable amount of different digital games, there is no agreement on how to best evaluate the player experience. Some of the most common questionnaires are the Player Experience of Need Satisfaction (PENS) [17] and the Game Experience Questionnaire (GEQ) [13]. However, while past work has raised evidence for the adequacy of the PENS to be used in research, the underlying factor structure of the GEQ is susceptible to critique [3, 8]. Azadvar and Canossa [2] developed the Ubisoft Perceived Experience Questionnaire (UPEQ) with the intent to enable the creation of surveys to capture the subtle nuances of the player experience. In their validation study, they suggest that the subscales of the UPEQ correlate, among other things, with enjoyment, game recommendation, and rating and could be a reliable and consistent assessment tool. Given the research community's need for more appropriate questionnaires, the UPEQ was quickly accepted as an alternative to other existing player experience questionnaires [18]. This paper aims to support the use of appropriate questionnaires in player experience research and sets out to further validate the UPEQ. To this end, an online survey asking about the player experience for League of Legends (LoL) [14], a popular Multiplayer Online Battle Arena (MOBA) game, was conducted, with the intent to answer the question of whether the proposed factor structure can be supported in this data. LoL has been chosen as a game to base this research on due to it being one of the most played online games to date with a vast online community. ${ }^{1}$ Previous research has shown that Self-determination Theory-based questionnaires, such as the PENS [10] or the User Motivation Inventory [4], are useful to study players' experience in LoL. In addition to the validation of the UPEQ, we were interested to see if we could find empirical support for the suggested correlation of the UPEQ subscales with video game enjoyment.

\section{METHOD}

\subsection{Measures}

2.1.1 Ubisoft Perceived Experience Questionnaire (UPEQ). The UPEQ was developed by Azadvar and Canossa and is based on the Selfdetermination Theory (SDT) $[6,15]$. The questionnaire includes subscales for the basic psychological needs of Autonomy, Competence and Relatedness. To develop the items included in this questionnaire, the authors drew inspiration from several need satisfaction scales,

\footnotetext{
${ }^{1} 100$ million monthly active users in 2016 https://www.statista.com/statistics/317099/ number-lol-registered-users-worldwide/ (viewed: 27. Aug 2021) and the second most popular PC games worldwide in December 2020 https://www.statista.com/statistics/ 1227532/top-pc-games-monthly-active-users/ (viewed: 27. Aug 2021)
} 
most notably from the Basic Need Satisfaction Scale - In General by Ilardi et al. [7]. With this procedure, the UPEQ was designed to measure above mentioned basic needs with a total of 21 items, 6 items each for the subscales Autonomy and Competence and 9 items for the subscale Relatedness. In our study, the items were rated on a 5-point Likert-scale ranging from "1 - Strongly disagree" to "5 Strongly agree".

2.1.2 Intrinsic Motivation Inventory (IMI). In line with Mekler et al. we define game enjoyment as "[...] the positive cognitive and affective appraisal of the game experience, and [game enjoyment] may in part be associated with the support of player needs and values" [9, p. 1]. Based on this definition, which includes player needs as a possible correlate, we chose to use the subscale Interest/Enjoyment from the Intrinsic Motivation Inventory (IMI) [16] to measure game enjoyment. The subscale Interest/Enjoyment consists of seven items assessed on a 7-point Likert-scale ranging from "1 - Strongly disagree" to "7 - Strongly agree". Like the PENS and UPEQ, the IMI is based on SDT, and the subscale Interest/Enjoyment has been described as the most widely used standardized questionnaire for measuring game enjoyment in video game research [9]. Therefore, because the UPEQ is intended to predict enjoyment and the IMI is anchored in the same theoretical framework (i.e., SDT), it was selected as an appropriate scale for the present study.

\subsection{Procedure}

Three hundred and ninety-seven participants took part in an Englishlanguage online survey published on a subpage of the public website Reddit (https://www.reddit.com/r/leagueoflegends/). During this survey, the participants were asked about their experience with the video game League of Legends (LoL) by game developer Riot Games [14]. It is to note here that the above-mentioned subreddit is specifically for the said video game. Therefore, all participants had at least some exposure to or experience with the game. After answering multiple questions about gaming habits and in-game demographics, such as in-game rank and the amount of time played, participants were asked to assess their player experience with the UPEQ. Additionally, they had to evaluate their enjoyment when playing the game with the subscale Interest/Enjoyment of the IMI. Finally, basic demographic questions were presented as well as the option to give feedback about the survey. Participation in the survey was voluntary, and the participants received no compensation for their time.

\subsection{Participants}

From the initial 397 responses, 369 (324 male, 32 female, 6 nonbinary, and 7 that preferred not to answer) remained after data cleaning. Data cleaning consisted of the elimination of participants under 18, participants who failed to answer an attention check item correctly, and participants who had self-advised not to use their data for data quality reasons. The participants age ranged from 18 to $44(M=22.63, S D=3.9)$. In contrast to the study of Azadvar and Canossa, no information on nationality or language proficiency was collected in this study. Every in-game rank was represented at least once within the present data with the highest number of players in Gold $(n=88)$ and Platinum $(n=76)$ tier, while 65 participants were not ranked at all.

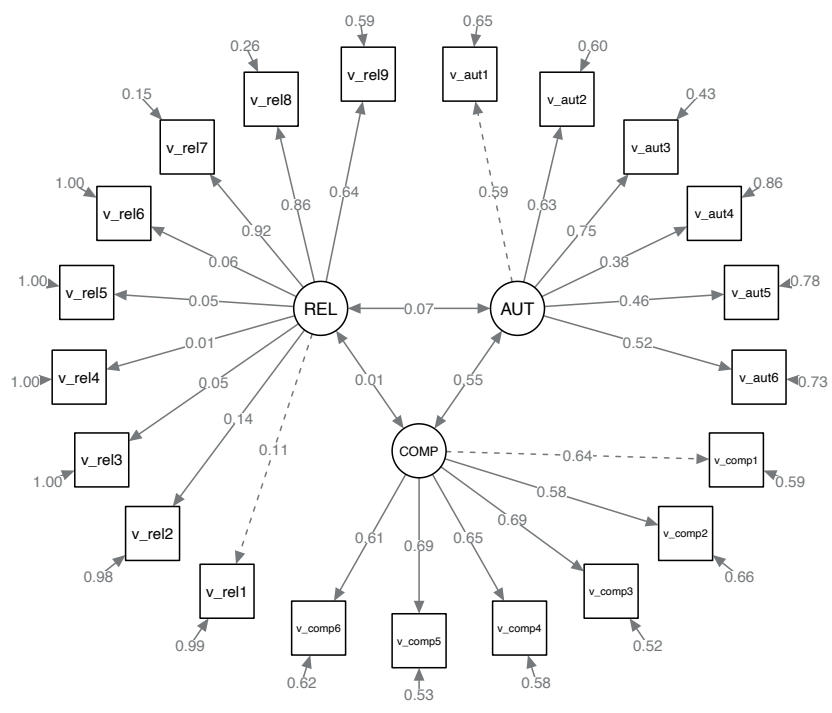

Figure 1: Measurement model of the original UPEQ with standardized loadings.

\section{RESULTS}

\subsection{Confirmatory Factor Analysis (CFA)}

First, a CFA was used to examine the model fit of the 3-factor model of the UPEQ proposed by Azadvar and Canossa [2] as a test of construct validity. Since the normality assumption is violated for our data, MLR was chosen as an estimator. This resulted in unacceptable fit indices $\left(\chi^{2}(186)=1116.2, p<0.01 ; \chi^{2} / d f=6\right.$; $C F I=0.627 ; T L I=0.579 ; R M S E A=0.116 ; S R M R=0.125)$. Results showed that only the factors of autonomy and competence covary significantly with each other $(p=0.02)$ while relatedness showed no significant covariance with either one of them. Inspection of standardized loadings revealed substantial differences for the factor relatedness, with values ranging from 0.01 up to 0.95 (see Figure 1).

\subsection{Regression Analysis}

As described in the development study, a beneficial characteristic of the questionnaire is the independent correlation of its subscales with enjoyment [2, p. 2, Table 1]. Therefore, this relationship was used to evaluate the criterion validity of the original questionnaire. A multiple linear regression was calculated to assess the prediction of game enjoyment, measured with the subscale Interest/Enjoyment of the IMI, through the subscales of the UPEQ, Autonomy, Competence and Relatedness. For this analysis, the means of the subscales were calculated for each participant. A significant regression equation was found $(F(3,365)=74.15, p<0.01)$, with an $R^{2}=0.37$. Participants' predicted Interest/Enjoyment is equal to $1.08+0.63 \mathrm{Au}$ tonomy +0.14 Competence +0.4 Relatedness. With the present data, only Autonomy $(b=0.63, t(365)=9.26, p<0.01)$ and Relatedness $(b=0.4, t(365)=5.6, p<0.01)$ acted as significant predictors for player's enjoyment but not Competence $(b=0.14, t(365)=1.96$, $p=0.05)$. 
Table 1: Factor loadings $>.25$ of the UPEQ items with the original 3-factor model.

\begin{tabular}{|c|c|c|c|c|}
\hline Item name & Item & PA1 & PA3 & PA2 \\
\hline v_aut1 & I was free to decide how I wanted to play. & & 0.55 & \\
\hline v_aut2 & I could approach League of Legends in my own way. & & 0.51 & \\
\hline v_aut3 & The game allowed me to play the way I wanted to. & & 0.72 & \\
\hline v_aut4 & I had important decisions to make when playing. & 0.49 & & \\
\hline v_aut5 & The choices I made while playing influenced what happened. & 0.35 & 0.26 & \\
\hline v_aut6 & My actions had an impact on the game. & 0.39 & 0.28 & \\
\hline V_comp1 & With time, I became better at playing. & 0.63 & & \\
\hline v_comp2 & My gaming abilities have improved since the beginning. & 0.55 & & \\
\hline v_comp3 & My mastery of the game improved with practice. & 0.69 & & \\
\hline v_comp4 & I was good at playing. & 0.56 & & \\
\hline v_comp5 & I felt competent at playing. & 0.60 & & \\
\hline v_comp6 & I felt very capable and effective when playing. & 0.48 & 0.29 & \\
\hline v_rel1 & I really like the people I play with. & & 0.52 & \\
\hline v_rel2 & I consider players I regularly interact with to be my friends. & & 0.33 & \\
\hline v_rel3 & Other players are friendly towards me. & & 0.57 & \\
\hline v_rel4 & What other players did in the game had an impact on my actions. & 0.43 & & \\
\hline v_rel5 & I had to adapt my actions to other players' actions. & 0.45 & & \\
\hline v_rel6 & I was paying attention to other players' actions. & 0.52 & & \\
\hline v_rel7 & I felt close to some of the characters. & & & 0.92 \\
\hline v_rel8 & I was bonding with some of the characters. & & & 0.84 \\
\hline v_rel9 & I cared about what happens to some of the characters. & & & 0.65 \\
\hline
\end{tabular}

\subsection{Exploratory Factor Analysis (EFA)}

To examine the factor structure of the questionnaire and explain the non-optimal fit of the 3-factor model, an EFA was conducted. The Kaiser-Meyer-Olkin (KMO) measure of sampling adequacy was acceptable with a score of .77 and the Bartlett's test of sphericity was significant $\left(\chi^{2}(210)=2634, p<.001\right)$. The EFA showed that the items do not load on the factors in the way the authors intended them to (see Table 1). It also provided evidence that the 3-factor model does not fit the data well. In the 3-factor model, 12 items loaded onto factor one (loading of $>0.3$ ), accounting for $15.6 \%$ of variance, while factor 2 (3 items) explained $9.8 \%$ and factor 3 (6 items) explained $10.3 \%$ of variance, resulting in a cumulative explained variance of $35.7 \%$.

Next, parallel analysis was used to determine the number of factors that best explain the present data. This analysis revealed 6 factors, which was supported by an analysis of Bayesian Information Criterion (BIC). With the BIC adjusted to the sample size, it supported a 7-factor model. To verify these findings, two new EFAs for a 6- and a 7-factor model were calculated. The 6-factor model showed a cumulative explained variance of $47.1 \%$ with bundles of 3 items loading onto the different factors. Only the items 4-6 of the subscale Relatedness did not load onto a factor on their own but loaded weakly onto factor 1, together with the items 1-3 of the subscale Competence, and onto factor 6, together with the items 4-6 of the subscale Autonomy (see Table 2). Using the 7-factor model, the cumulative explained variance increased to $50.2 \%$, with the above-mentioned items 4-6 of the subscale Relatedness now solely loading on the newly added 7. factor (see Table 3).
Table 2: Factor loadings $>.25$ of the UPEQ items with the 6factor model.

\begin{tabular}{|c|c|c|c|c|c|c|}
\hline Item name & PA2 & PA4 & PA1 & PA3 & PA6 & PA5 \\
\hline v_aut1 & & & & 0.57 & & \\
\hline v_aut2 & & & & 0.66 & & \\
\hline v_aut3 & & & & 0.80 & & \\
\hline v_aut4 & & & & & 0.46 & \\
\hline v_aut5 & & & & & 0.69 & \\
\hline v_aut6 & & & & & 0.56 & \\
\hline v_comp1 & & & 0.76 & & & \\
\hline v_comp2 & & & 0.50 & & & \\
\hline v_comp3 & & & 0.73 & & & \\
\hline v_comp4 & & 0.84 & & & & \\
\hline v_comp5 & & 0.75 & & & & \\
\hline v_comp6 & & 0.61 & & & & \\
\hline v_rel1 & & & & & & 0.76 \\
\hline v_rel2 & & & & & & 0.66 \\
\hline v_rel3 & & & & & & 0.39 \\
\hline v_rel4 & & & 0.36 & & 0.37 & \\
\hline v_rel5 & & & 0.31 & & 0.34 & \\
\hline v_rel6 & & & 0.26 & & 0.30 & \\
\hline v_rel7 & 0.93 & & & & & \\
\hline v_rel8 & 0.85 & & & & & \\
\hline v_rel9 & 0.65 & & & & & \\
\hline
\end{tabular}


Table 3: Factor loadings $>.25$ of the UPEQ items with the 7factor model.

\begin{tabular}{llllllll}
\hline Item name & PA2 & PA4 & PA1 & PA3 & PA5 & PA6 & PA7 \\
\hline v_aut1 & & & & 0.60 & & & \\
v_aut2 & & & & 0.56 & & & \\
v_aut3 & & & & 0.94 & & & \\
v_aut4 & & & & & 0.34 & & \\
v_aut5 & & & & & 0.74 & & \\
v_aut6 & & & & & 0.74 & & \\
v_comp1 & & & 0.86 & & & & \\
v_comp2 & & & 0.56 & & & & \\
v_comp3 & & & 0.73 & & & & \\
v_comp4 & & 0.86 & & & & & \\
v_comp5 & & 0.74 & & & & & \\
v_comp6 & & 0.60 & & & & & \\
v_rel1 & & & & & & 0.77 & \\
v_rel2 & & & & & & 0.65 & \\
v_rel3 & & & & & & 0.37 & \\
v_rel4 & & & & & & & 0.65 \\
v_rel5 & & & & & & & 0.51 \\
v_rel6 & & & & & & & 0.36 \\
v_rel7 & 0.93 & & & & & & \\
v_rel8 & 0.86 & & & & & & \\
v_rel9 & 0.64 & & & & & & \\
\hline
\end{tabular}

\section{DISCUSSION}

Confirmatory factor analysis showed that the 3-factor model proposed by the authors of the UPEQ could not be supported by our data. Results show inconsistencies in every factor, but especially in the subscale Relatedness. An exploratory factor analysis suggests that not all of the items load onto the factors proposed by the authors. Instead, they load on different or even multiple factors. These cross-loadings lead to a low percentage of explained variance by the model. The wording of the items explains these results: there are thematic differences in the questions underlying the same subscales (see Table 1). The items seem to be grouped as triplets which ask about different aspects of a video game, even though they exist in the subscale. These findings are supported by the EFA results for different factor models. With enough factors to load onto, the items group themselves as the triplets mentioned above under the factors. This leads to a higher percentage of explained variance by both the 6- and 7-factor models. The latter has the clearest assignment of variables to the factors and the highest percentage of explained variance. Although the findings of this study report evidence that the UPEQ has an insufficient model fit when modeled with three factors, this does not mean that the questionnaire can not be a valid predictor of player experience and the commercial success of a video game. The regression analysis showed that both Autonomy and Relatedness are significant predictors for players' game enjoyment. However, it is questionable whether the proposed subscales measure what the authors intended them to measure. This complicates the interpretation of data provided by studies using the UPEQ.

\subsection{Limitations and Next Steps}

One limitation of the current work is that there was no question regarding when the participants lastly played League of Legends. Although all participants reported to have played to game at some point, this doesn't directly compare to the work of Azardvar and colleagues where they asked about a game the participants played at that time of the survey. Additionally, it is to mention that a relatively large amount of the participants have not played the ranked mode of LoL for some time and therefore stated their rank as Unranked. While this gives no indication about their in-game playtime, the argument could be made that ranked games do provide a different experience than playing unranked. Additionally, in this work-in-progress, we did not consider factor structures with multiple layers, such as the existence of a single underlying factor accounting for correlations between the factors. The use of bifactor analysis was recently recommended for use in PX research to investigate such structures and could therefore be considered in future research investigating the UPEQ [5]. Lastly, we did not analyze the questionnaire on a semantic level. While we found evidence that the proposed factor model does not work as intended, future research needs to have a close look at the content of the items of the UPEQ and reevaluate their affiliation to the different subscales. For example, studies using the PENS sometimes choose not to collect data for the subscale Relatedness when testing for a non-multiplayer game [3]. At the same time, the UPEQ combines interactions with other players and relationships to non-player characters (NPCs) in the same subscale. In a next step, we plan to conduct item analysis to identify if items of the UPEQ should be rephrased or excluded. We also plan to include data from players' experience with other games than LoL for this step. Additionally, we consider running confirmatory factor analysis to examine the model fit for the 6- and 7-factor models and the models' construct validity. We would also run regression analysis for these new models of the UPEQ to assess their ability to predict enjoyment as a test of criterion validity. In case one or both of the factor models proposed by this paper show an adequate fit, the UPEQ could then be compared to the PENS and other player experience scales such as the Player Experience Inventory [1] or the Game User Experience Satisfaction Scale [12] in terms of their prediction of enjoyment and in-game behavior.

\section{CONCLUSION}

This paper provides initial evidence against the proposed 3-factor model of the Ubisoft Perceived Experience Questionnaire and proposes a 6- or 7-factor model when using all 21 items of the questionnaire. Despite the insufficient model fit of the 3-factor model, a regression analysis revealed that the questionnaire could still predict game enjoyment, as both the subscales Autonomy and Relatedness acted as significant predictors. As a next step, we plan to continue with the evaluation of the UPEQ, with particular attention to the wording of the items for the construct Relatedness. We also plan to investigate the stability of the proposed 6- and 7-factor models in the context of other digital games.

\section{DATA AVAILABILITY STATEMENT}

Data, analysis scripts, and supplementary materials for this study are available on OSF: https://osf.io/6nhts/. 


\section{REFERENCES}

[1] Vero Vanden Abeele, Katta Spiel, Lennart Nacke, Daniel Johnson, and Kathrin Gerling. 2020. Development and validation of the player experience inventory: A scale to measure player experiences at the level of functional and psychosocial consequences. International Journal of Human-Computer Studies 135 (2020), 102370. https://doi.org/10.1016/j.ijhcs.2019.102370

[2] Ahmad Azadvar and Alessandro Canossa. 2018. UPEQ: Ubisoft Perceived Experience Questionnaire: A Self-Determination Evaluation Tool for Video Games. In Proceedings of the 13th International Conference on the Foundations of Digital Games (Malmö, Sweden) (FDG '18). Association for Computing Machinery, New York, NY, USA, Article 5, 7 pages. https://doi.org/10.1145/3235765.3235780

[3] Florian Brühlmann and Gian-Marco Schmid. 2015. How to Measure the Game Experience? Analysis of the Factor Structure of Two Questionnaires. In Proceedings of the 33rd Annual ACM Conference Extended Abstracts on Human Factors in Computing Systems (Seoul, Republic of Korea) (CHI EA '15). Association for Computing Machinery, New York, NY, USA, 1181-1186. https: //doi.org/10.1145/2702613.2732831

[4] Florian Brühlmann, Philipp Baumgartner, Günter Wallner, Simone Kriglstein, and Elisa D. Mekler. 2020. Motivational Profiling of League of Legends Players. Frontiers in Psychology 11 (2020), 1307. https://doi.org/10.3389/fpsyg.2020.01307

[5] Paul Cairns and Christopher Power. 2018. Measuring Experiences. In New Directions in Third Wave Human-Computer Interaction: Volume 2 - Methodologies, Michael Filimowicz and Veronika Tzankova (Eds.). Springer International Publishing, Cham, 61-80. https://doi.org/10.1007/978-3-319-73374-6_5

[6] Edward L Deci and Richard M Ryan. 2004. Handbook of self-determination research University Rochester Press, Rochester, NY, USA.

[7] Barbara C. Ilardi, Dean Leone, Tim Kasser, and Richard M. Ryan. 1993. Employee and Supervisor Ratings of Motivation: Main Effects and Discrepancies Associated with Job Satisfaction and Adjustment in a Factory Setting1. Fournal of Applied Social Psychology 23, 21 (1993), 1789-1805. https://doi.org/10.1111/j.1559-1816. 1993.tb01066.x

[8] Effie L.-C. Law, Florian Brühlmann, and Elisa D. Mekler. 2018. Systematic Review and Validation of the Game Experience Questionnaire (GEQ) - Implications for Citation and Reporting Practice. In Proceedings of the 2018 Annual Symposium on Computer-Human Interaction in Play (Melbourne, VIC, Australia) (CHI PLAY
'18). Association for Computing Machinery, New York, NY, USA, 257-270. https: //doi.org/10.1145/3242671.3242683

[9] Elisa D. Mekler, Julia Ayumi Bopp, Alexandre N. Tuch, and Klaus Opwis. 2014. A Systematic Review of Quantitative Studies on the Enjoyment of Digital Entertainment Games. In Proceedings of the SIGCHI Conference on Human Factors in Computing Systems (Toronto, Ontario, Canada) (CHI '14). Association for Computing Machinery, New York, NY, USA, 927-936. https://doi.org/10.1145/2556288.2557078

[10] Marçal Mora-Cantallops and Miguel Ángel Sicilia. 2018. Exploring player experience in ranked League of Legends. Behaviour \& Information Technology 37, 12 (2018), 1224-1236. https://doi.org/10.1080/0144929X.2018.1492631

[11] Mary Beth Oliver, Nicholas David Bowman, Julia K Woolley, Ryan Rogers, Brett I Sherrick, and Mun-Young Chung. 2016. Video games as meaningful entertainment experiences. Psychology of Popular Media Culture 5, 4 (2016), 390. https://doi. org/10.1037/ppm0000066

[12] Mikki H. Phan, Joseph R. Keebler, and Barbara S. Chaparro. 2016. The Development and Validation of the Game User Experience Satisfaction Scale (GUESS). Human Factors 58, 8 (2016), 1217-1247. https://doi.org/10.1177/0018720816669646 arXiv:https://doi.org/10.1177/0018720816669646 PMID: 27647156

[13] Karolien Poels, YAW De Kort, and Wijnand A IJsselsteijn. 2007. D3. 3: Game Experience Questionnaire: development of a self-report measure to assess the psychological impact of digital games.

[14] Riot Games. 2009. League of Legends. Game [PC]. Riot Games, Los Angeles, CA, USA.

[15] Richard M Ryan and Edward L Deci. 2017. Self-determination theory: Basic psychological needs in motivation, development, and wellness. Guilford Publications, New York City, NY, USA.

[16] Richard M Ryan, Valerie Mims, and Richard Koestner. 1983. Relation of reward contingency and interpersonal context to intrinsic motivation: A review and test using cognitive evaluation theory. Fournal of personality and Social Psychology 45, 4 (1983), 736. https://doi.org/10.1037/0022-3514.45.4.736

[17] Richard M Ryan, C Scott Rigby, and Andrew Przybylski. 2006. The Motivational Pull of Video Games: A Self-Determination Theory Approach. Motivation and Emotion 30, 4 (2006), 344-360. https://doi.org/10.1007/s11031-006-9051-8

[18] April Tyack and Elisa D. Mekler. 2020. Self-Determination Theory in HCI Games Research: Current Uses and Open Questions. Association for Computing Machinery, New York, NY, USA, 1-22. https://doi.org/10.1145/3313831.3376723 